\title{
Repetitive Transcranial Magnetic Stimulation Enhances Recovery in Central Cord Syndrome Patients
}

\author{
Hana Choi, MD', Kyung Cheon Seo, $\mathrm{MD}^{1}$, Tae Uk Kim, MD, PhD ${ }^{1}$, \\ Seong Jae Lee, $\mathrm{MD}^{1}$, Jung Keun Hyun, $\mathrm{MD}, \mathrm{PhD}^{1,2,3,4}$

\begin{abstract}
${ }^{1}$ Department of Rehabilitation Medicine, Dankook University College of Medicine, Cheonan; ${ }^{2}$ Department of Nanobiomedical Science and BK21 Plus NBM Global Research Center for Regenerative Medicine, Dankook University, Cheonan; ${ }^{3}$ Institute of Tissue Regeneration Engineering (ITREN), Dankook University, Cheonan; ${ }^{4}$ Wiregene Co. Ltd., Cheonan, Korea
\end{abstract}

Objective To investigate the effect of repetitive transcranial magnetic stimulation (rTMS) on neurological and functional recovery in patients with central cord syndrome (CCS) involving the upper extremities between the treated and non-treated sides of the treated group and whether the outcomes are comparable to that of the untreated control group.

Methods Nineteen CCS patients were treated with high-frequency $(20 \mathrm{~Hz})$ rTMS over the motor cortex for 5 days. The stimulation side was randomly selected, and all the subjects received conventional occupational therapy during the rTMS-treatment period. Twenty CCS patients who did not receive rTMS were considered as controls. Clinical assessments, including those by the International Standard for Neurological Classification of Spinal Cord Injury, the Jebsen-Taylor Hand Function Test, and the O'Connor Finger Dexterity Test were performed initially and followed up for 1 month after rTMS treatment or 5 weeks after initial assessments.

Results The motor scores for upper extremities were increased and the number of improved cases was greater for the treated side in rTMS-treated patients than for the non-treated side in rTMS-treated patients or controls. The improved cases for writing time and score measured on the Jebsen-Taylor Hand Function Test were also significantly greater in number on the rTMS-treated side compared with the non-treated side and controls. There were no adverse effects during rTMS therapy or the follow-up period.

Conclusion The results of the application of high-frequency rTMS treatment to CCS patients suggest that rTMS can enhance the motor recovery and functional fine motor task performance of the upper extremities in such individuals.

Keywords Spinal cord injuries, Central cord syndrome, Transcranial magnetic stimulation, Upper extremity, Writing

Department of Rehabilitation Medicine, Dankook University College of Medicine, 119 Dandae-ro, Dongnam-gu, Cheonan 31116, Korea. Tel: +82-41550-6640, Fax: +82-41-551-7062, E-mail: rhhyun@dankook.ac.kr

ORCID: Hana Choi (http://orcid.org/0000-0002-5732-2903); Kyung Cheon Seo (http://orcid.org/0000-0003-4994-2210); Tae Uk Kim (http://orcid. org/0000-0001-9904-8781); Seong Jae Lee (http://orcid.org/0000-0001-7867-4695); Jung Keun Hyun (http://orcid.org/0000-0001-9254-4424).

(c) This is an open-access article distributed under the terms of the Creative Commons Attribution Non-Commercial License (http://creativecommons.org/ licenses/by-nc/4.0) which permits unrestricted noncommercial use, distribution, and reproduction in any medium, provided the original work is properly cited. Copyright $\odot 2019$ by Korean Academy of Rehabilitation Medicine 


\section{INTRODUCTION}

Spinal cord injury (SCI) is a major devastating lesion and the treatments for the condition in the clinical setting remain controversial. A number of clinical trials aiming to achieve functional recovery in SCI patients have been carried out and most of the results relevant to the protection of the injured spinal cord and modulation of secondary injury following SCI were uncertain [1]. Recently, many studies have aimed to explore means of regenerating damaged neurons using various types of stem cells [2,3], regeneration-associated genes [4], biomaterials [5] or their combinations [6]. However, the reported treatment methods are invasive with effects limited for clinical application and many safety issues have not been considered in the existing studies. Another treatment strategy for post-SCI functional restoration is neuronal plasticity enhancement. Although neuronal plasticity after SCI is not as well studied or understood as that after brain lesion, some studies have reported collateral sprouting of spared axons to disconnected tracts and cortical remapping in incomplete SCI animal models $[7,8]$. In fact, in the case of extensive and proper plasticity of spared axons within the spinal cord, functional recovery could be expected $[9,10]$.

Repetitive transcranial magnetic stimulation (rTMS) has been reported to modulate cortical and spinal cord circuits and neuronal plasticity [11] to the extent of enhancing neuronal plasticity, which in turn could promote functional improvements after SCI [12]. Recent clinical studies have revealed that rTMS can reduce neuropathic pain [13] and spasticity [14] in SCI patients; however, the reported effects are variable and limited [15].

Central cord syndrome (CCS) is the most common type of SCI with frequent occurrence in older patients with cervical spondylosis or hyperextension injury [16]. CCS is characterized by upper extremity weakness that is disproportionately greater than that in the lower extremities [17]. Globally, the frequency of incidence of CCS, one of the major age-related spinal disorders [18] is on a constant rise with an aging population. Although the prognosis of CCS patients is usually better compared to the patients with other types of incomplete SCI, complete recovery is rare, and in fact, most of the CCS patients suffer from neuropathic pain and upper extremity dysfunction for the rest of their lives.
In the present study, subsequent to prospective application of rTMS to CCS patients, we aimed to delineate its effect on neurological and functional recovery of the upper extremities between the treated and non-treated sides of a treated group and determined whether the effect is comparable with the untreated control group.

\section{MATERIALS AND METHODS}

\section{Subjects}

We performed a prospective clinical trial with subacute stage (9-83 days post-injury) CCS patients recruited from the inpatient population in Department of Rehabilitation Medicine at Dankook University Hospital. The diagnostic criterion for CCS was similar to that of Schneider et al. [19]: SCI patients with disproportionately more motor impairment of the upper than the lower extremities. Any subjects having the following conditions were excluded: pre-existing or combined brain lesions, combined peripheral nerve injuries or polyneuropathies, metal implants in the head, history of substance abuse, severe cognitive dysfunction, and personal or family history of seizure. Nineteen patients with CCS (mean age, 53 years; 15 males and 4 females) participated in this study, and 20 CCS patients who disagreed to receive rTMS treatment were included as a control (mean age, 55 years; 19 males and 1 female). The most common neurological level of injury (NLI) was C5 (36.8\% in rTMS-treated patients and $40 \%$ in controls), and the severity of impairment in most of the subjects, according to the International Standards for the Neurological Classification of Spinal Cord Injury (ISNCSCI), was AIS (American Spinal Injury Association [ASIA] Impairment Scale) D (84.2\% in rTMS-treated patients and $80 \%$ in controls) (Tables 1,2 ). There was no difference in age or in the gender distribution, initial NLI, and initial AIS between rTMS-treated patients and controls. This study was approved by the Dankook University Hospital Institutional Review Board (No. 2013-04-015).

\section{Treatments}

All the subjects received high-frequency $(20 \mathrm{~Hz})$ rTMS over the unilateral motor cortex by means of a Magstim Super Rapid ${ }^{2}$ magnetic stimulator (Magstim, Carmarthenshire, UK) with a 70-mm diameter figure-of-eight coil. The side for rTMS treatment (to which the data analyzer was blind) was randomly determined and the treat- 
Table 1. Baseline characteristics of subjects

\begin{tabular}{|lccc}
\hline \multicolumn{1}{c}{ Characteristic } & Control group (n=20) & rTMS group (n=19) & p-value \\
\hline Age (yr) & $54.85 \pm 14.4$ & $52.78 \pm 10.5$ & 0.725 \\
\hline Sex & & & 0.123 \\
\hline Male & 19 & 15 & \\
\hline Female & 1 & 4 & 0.719 \\
\hline Initial neurological level of injury & & & \\
\hline C3 & $4(20)$ & $3(15.8)$ & \\
C4 & $5(25)$ & $6(31.6)$ & \\
C5 & $8(40)$ & $2(36.8)$ & 0.588 \\
\hline C6 & $1(5)$ & $1(5.3)$ & \\
C7 & $2(10)$ & & \\
\hline Initial ASIA impairment scale & $2(10)$ & $16(84.2)$ & \\
\hline C & $18(90)$ & $25.0 \pm 10.6$ & \\
\hline D & - & & \\
\hline Duration from onset to rTMS treatment (day) & & & \\
\hline
\end{tabular}

Values are presented as mean \pm standard deviation or number (\%).

rTMS, repetitive transcranial magnetic stimulation; ASIA, American Spine Injury Association.

$\mathrm{p}$-values were obtained from the likelihood ratio test for categorical data or from Mann-Whitney U-test for continuous data.

ment was performed once per day for 5 consecutive days. Stimulation of the left or right primary motor cortex (M1) induced a response in the contralateral abductor pollicis brevis muscle, which manifested as a muscle twitch that was recorded as a motor-evoked potential (MEP) using surface electromyography. We defined the 'treated side' as the contralateral side of the stimulated motor cortex; accordingly, when the left motor cortex was stimulated, the 'treated side' was considered as the right side. Twosecond bursts of $20 \mathrm{~Hz}$ were applied over the hand area of the assigned unilateral motor cortex at inter-train intervals of 28 seconds, for a total of 1,800 pulses during 30 minutes. The intensity of the stimulation was set to $90 \%$ of the resting motor threshold, which was defined as the lowest intensity necessary for the evocation of an MEP response of $50 \mu \mathrm{V}$ in $50 \%$ of trials. Also, the subjects received conventional occupational therapies including strengthening exercises, training in the activities of daily living, and manual dexterity exercises for 30 minutes twice per day during their rTMS treatment.

\section{Neurological assessment}

All the subjects received physical examination along with the motor and sensory, NLI and AIS evaluations by ISNCSCI at admission and again at 4 weeks post-rTMS treatment. We scored the upper extremity motor and sensory statuses from C5 to T1 and the lower-extremity ones from L2 to S1. Also, hand strength was objectively evaluated using a hand dynamometer and pinch gauge to measure the grip strength and lateral and tip pinch strengths exerted by the thumb and index fingers in both the hands. Each test was performed three times, and the score was recorded as the average of three values [20]. For comparison of the treated and non-treated sides of rTMS treated patients, the motor, sensory, and muscle power of both the sides of the control patients were divided by half by adding the left and right measurements.

\section{Functional assessment}

The hand and finger functional assessments were determined using the Jebsen-Taylor hand function test (JHFT) and the O'Connor Finger Dexterity Test (OFDT; Lafayette Instrument Co., Lafayette, IN, USA) at admission and 4 weeks post-rTMS treatment. The JHFT provides quantitative measurements of standardized tasks in assessing the following 7 subsets of hand function: writing, turning over $3 \times 5$-inch cards, picking up small common objects, simulated feeding, stacking checkers, picking up large light objects, and picking up large heavy objects. The time to complete each subset was recorded and com- 


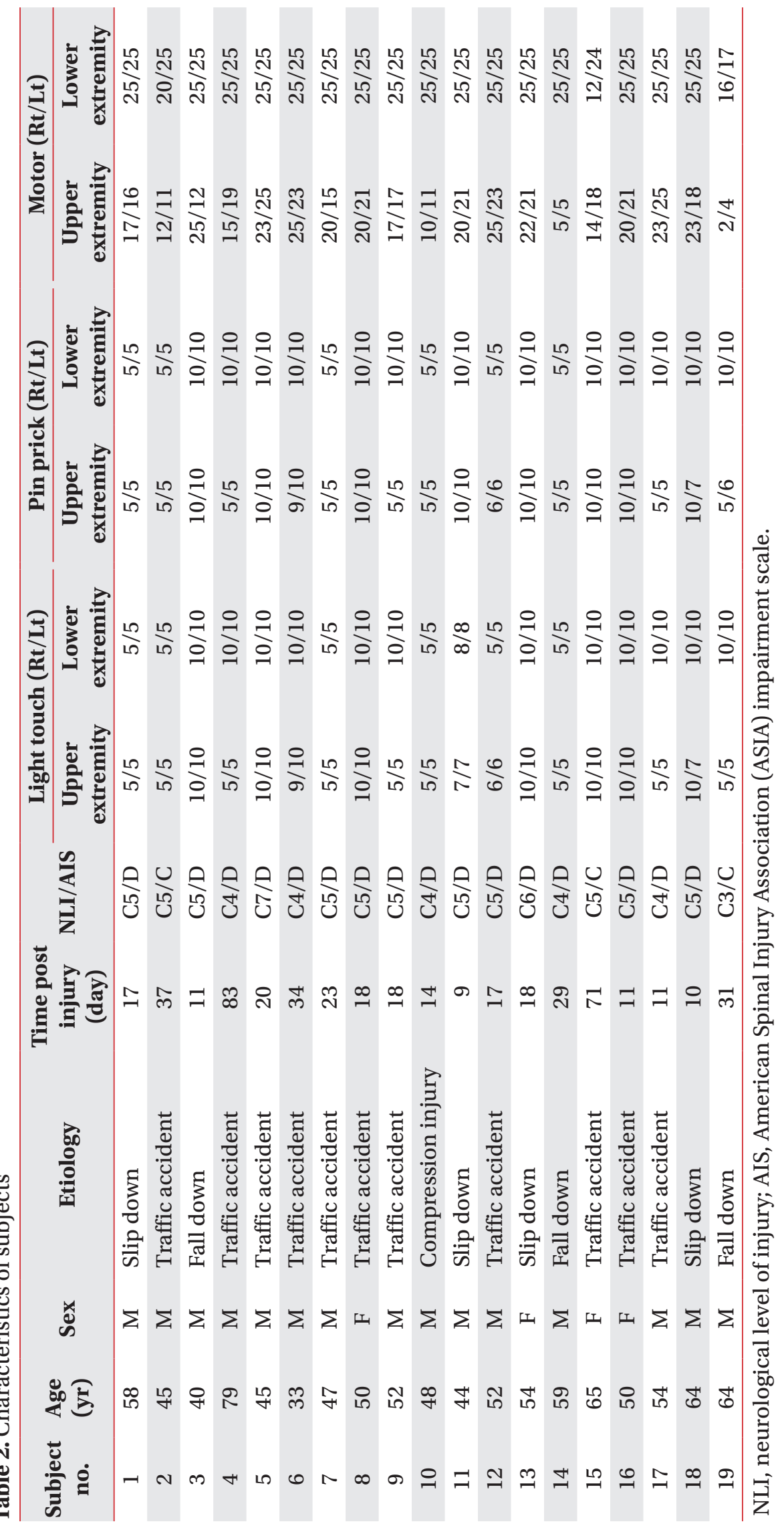




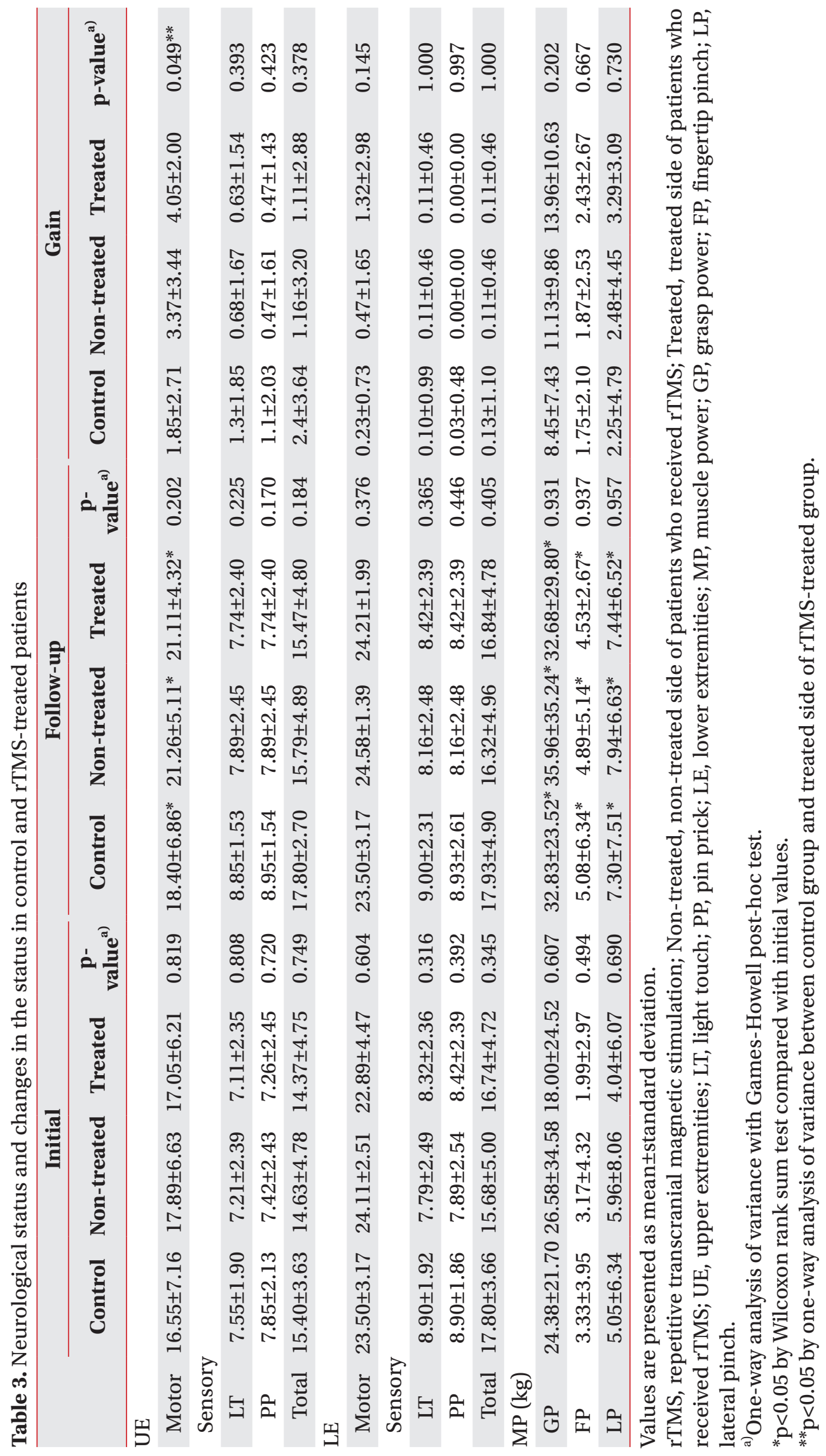


pared with the established normal value determined for age and sex. The OFDT is a standardized test used to evaluate rapid picking up and insertion of small pins into 100 holes arranged in 10 rows and spaced $1 / 2$ inch apart. The subjects were instructed to insert 3 pins per hole using their left or right hand. Following the guidelines in the manual, the time required by the subject to insert 3 pins into the first 5 rows of the board (total 50 holes) was considered to be the first halftime, and the time required to insert another 3 pins into the other 5 rows (total 50 holes) was considered as the second halftime. The raw score was recorded as the average of the first and second half times multiplied by 1.1. The standard score and percentile rank were determined according to the raw score ranges specified in the manual (Lafayette Instrument Co.). For comparison of the treated and non-treated sides of the rTMS treated patients, the results for the control patients were expressed as the averages of the left and right measurements.

\section{Statistical analysis}

Statistical analyses were performed using PASW Statistics version 23 (IBM Corp, Armonk, NY, USA). The Shapiro-Wilk test was used to reveal the normal distribution of the quantitative data obtained from the neurological and functional assessments. One-way analysis of variance and Games-Howell post-hoc test were performed to compare all the numerical data at the baseline and during the follow-up period among the treated and nontreated sides of the rTMS-treated patients and controls, and Wilcoxon rank sum test was employed to compare neurological and functional data between the initial and follow-up periods in the same subjects. The likelihood ratio test was used to analyze the subjects who showed any improvements following rTMS treatment on the treated and non-treated sides and controls. Spearman rank correlation analysis was performed to find relationships between the duration from onset to rTMS treatment and neurological and functional changes. The p-values less than 0.05 were considered to be significant.

\section{RESULTS}

Following randomization of the treated side, $11 \mathrm{pa}-$ tients received rTMS treatment on their right hemisphere (treated side, left) and 8 patients received treatment on their left hemisphere (treated side, right). There were no differences in the changes in neurological and functional status between the left- and right-side-treated subjects. No adverse effect was found during or after rTMS treatment in any of the subjects.

The upper extremity motor scores for rTMS-treated patients and controls were improved 4 weeks after rTMS treatment or 5 weeks after initial assessments, and notably, the gain score of both the sides of rTMS-treated patients exhibited more increase compared with the controls (gain score, $4.05 \pm 2.00$ and $3.37 \pm 3.44$ for both the sides of rTMS-treated patients vs. $1.85 \pm 2.71$ for controls; $\mathrm{p}=0.049$ ) (Table 3 ). In addition, when we counted the number of improved cases for each side of rTMS-treated patients and controls, there were more cases with the treated side than the non-treated side in rTMS-treated patients and controls (95\%, 68\%, and 65\%, respectively; $\mathrm{p}=0.035$ ) (Fig. 1A). Also, the grasp power and fingertip
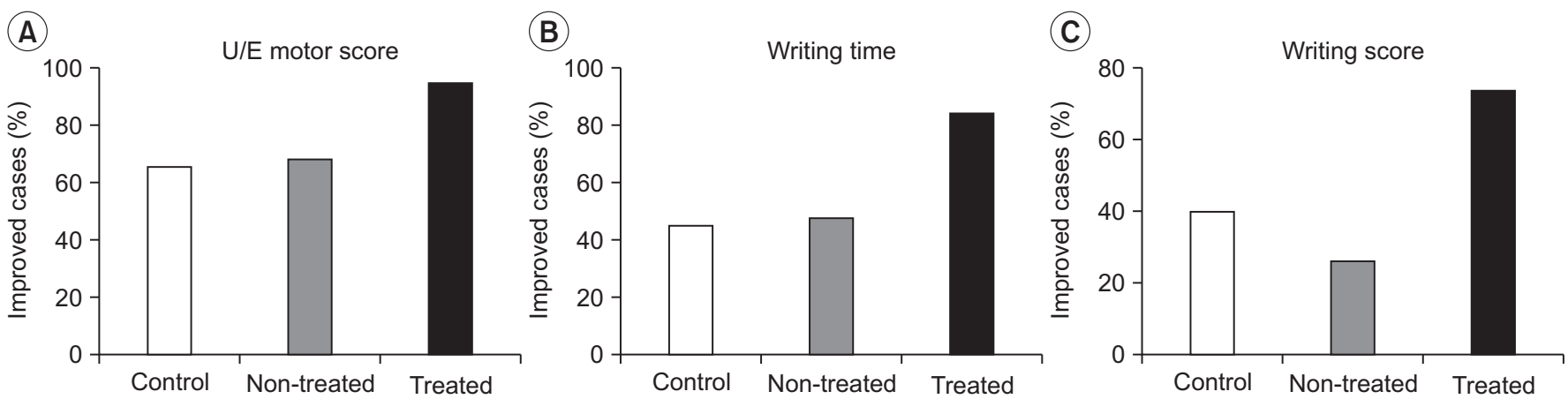

Fig. 1. Featured neurological and functional changes in control and rTMS-treated patients: number of subjects with improvement (\%) with respect to UE motor score (A), writing time (B) and score (C) by JHFT for controls or rTMS-treated patients of treated and non-treated sides during the follow-up period. All p-values were less than 0.05 by likelihood ratio test. rTMS, repetitive transcranial magnetic stimulation; UE, upper extremity; JHFT, Jebsen Hand Function Test. 


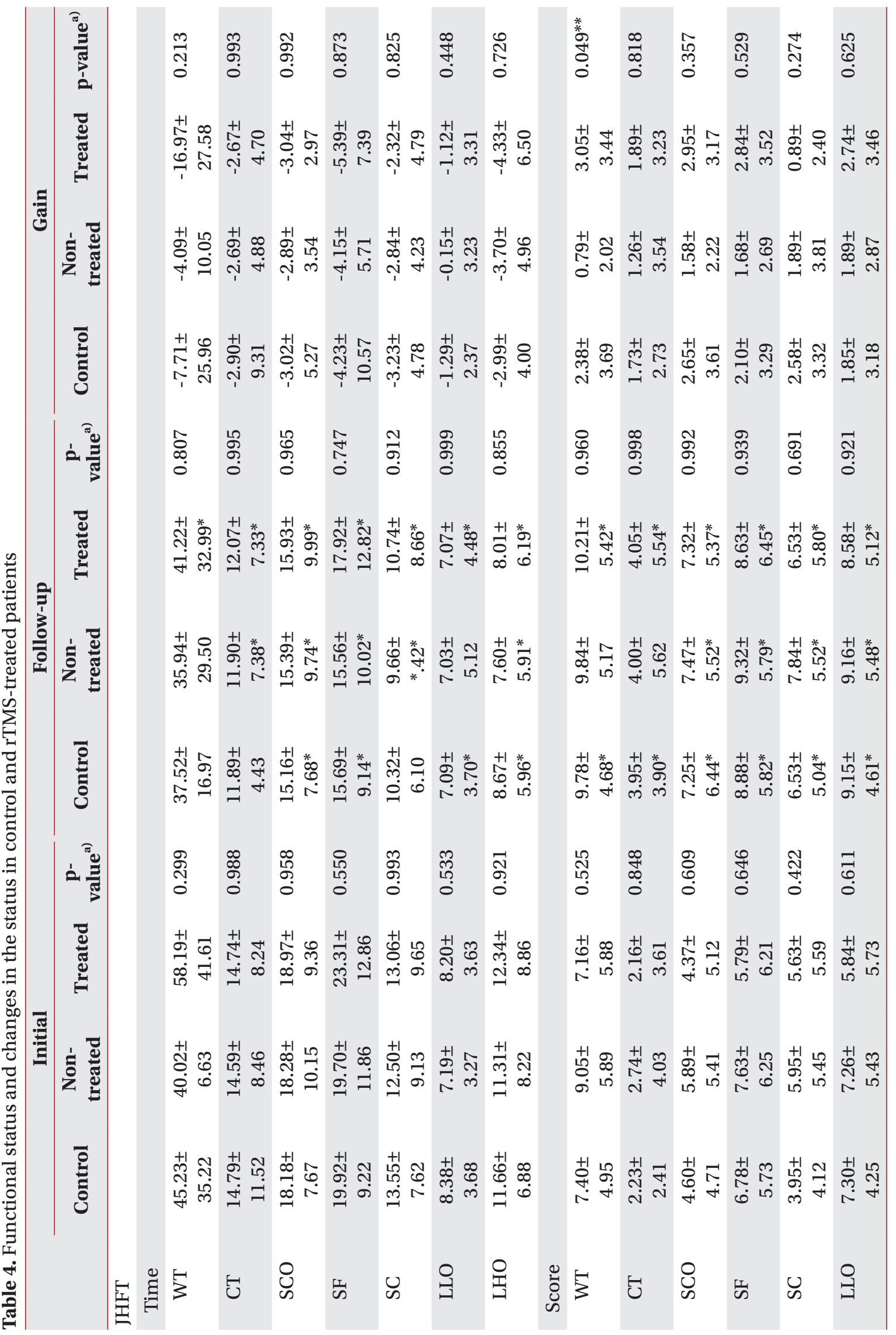




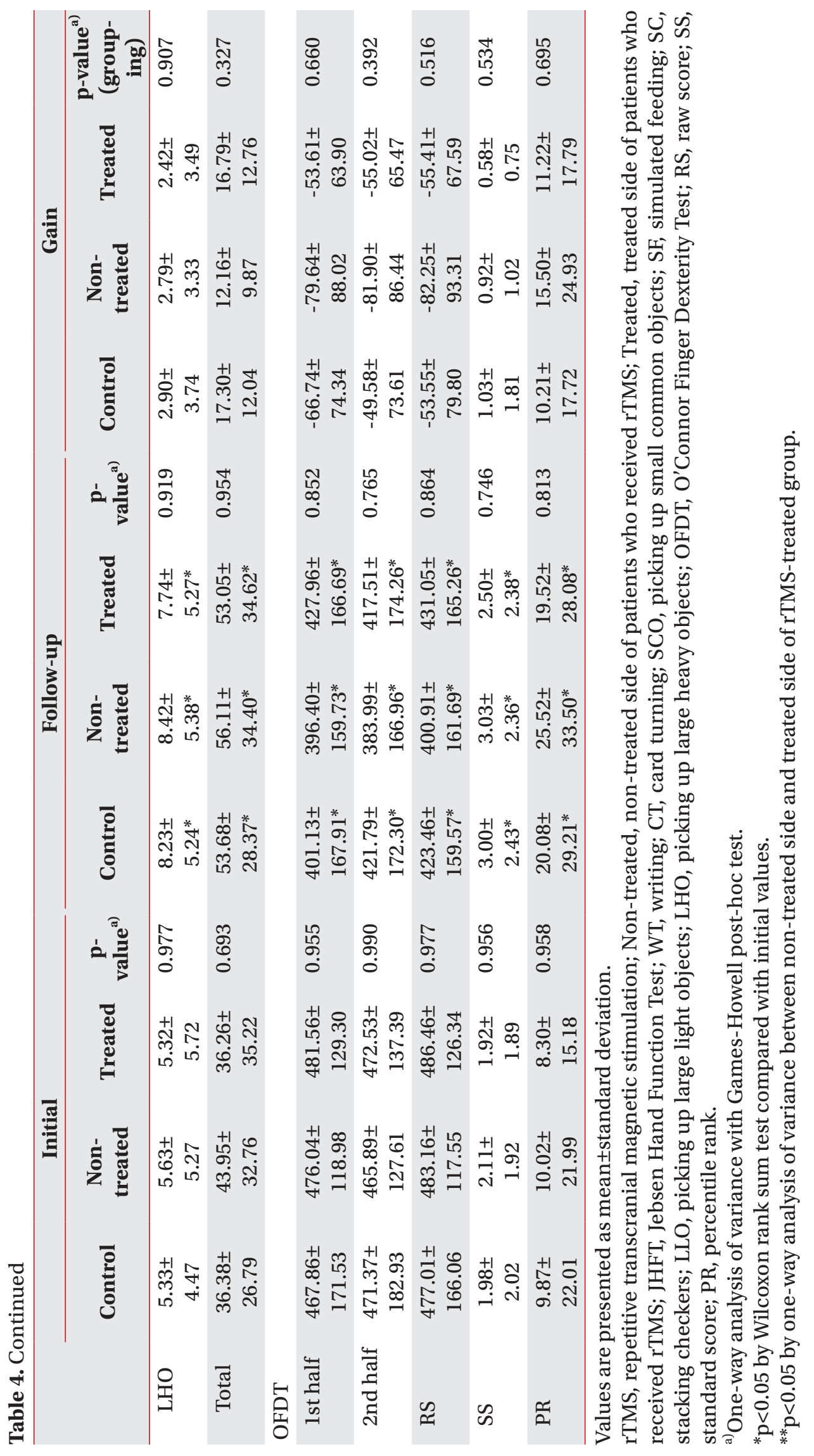


and lateral pinch powers were increased during the follow-up period, regardless of the side or rTMS treatment (Table 3). The sensory score of the upper extremities and the motor and sensory scores of the lower extremities exhibited no change on either side of rTMS-treated patients or controls during the follow-up period (Table 3 ).

The functional status measured by JHFT and OFDT exhibited improvement on the treated side in rTMS-treated cases and on the non-treated side in rTMS-treated patients. Neither the writing time score nor the card turning score measured by JHFT exhibited any change at 4 weeks after rTMS treatment, and time for writing, card turning, and picking up large heavy objects showed no improvement in controls (Table 4). When we compared the number of cases of improvement in writing time and score between the treated and non-treated sides in rTMS-treated patients and controls, more cases were observed on the treated side in rTMS-treated patients: $84 \%$ for the treated side vs. $47 \%$ for the non-treated side of rTMS-treated patients and $45 \%$ for controls, $\mathrm{p}=0.016 ; 74 \%$ for treated side vs. $26 \%$ for non-treated side of rTMS-treated patients and $40 \%$ for controls, $\mathrm{p}=0.009$, respectively (Fig. 1B, 1C).

The NLI changed to $15.8 \%$ (3 subjects) in rTMS-treated patients and $25 \%$ ( 5 subjects) in controls during the follow-up period without any statistical difference (Table 5). The AIS changed from C to D in 2 rTMS-treated patients and controls, and there was no relationship between duration from onset to rTMS treatment and neurological and functional changes in rTMS-treated patients (data not shown).

\section{DISCUSSION}

In the past, numerous experiments and clinical trials have been performed to investigate post-SCI functional restoration by modulation of inflammation and secondary injury, enhancement of axonal regeneration or remyelination or neuronal plasticity. Neuronal plasticity is represented by brain remapping, collateral sprouting of spared axons, and synaptic changes that allow neurons in the central nervous system to compensate for neuronal injury and to adjust their activity in response to new environments. Remapping of the motor cortex has been noticed subsequent to partial cervical spinal cord transection in mice [7]. Also, it has been observed that some injured motor tracts sprout collaterally and integrate into propriospinal circuits and even cross the midline bypassing the lesions after cervical spinal cord hemisection in rodents $[8,21]$. However, in most of the cases, spontaneous plasticity changes compensatory to SCI are not sufficient to achieve functional restoration following SCI. Stimulation of the brain or spinal cord might boost postSCI neuronal plasticity by enhancing the strength of the synaptic connections [22], to which end, task-specific re-

Table 5. Changes in the NLI in control and rTMS-treated patients

\begin{tabular}{|c|c|c|c|c|c|c|c|c|}
\hline \multirow{2}{*}{ Initial NLI } & \multicolumn{8}{|c|}{ Follow-up NLI } \\
\hline & C3 & C4 & $\mathbf{C 5}$ & C6 & C7 & $\mathbf{C 8}$ & T1 & Total \\
\hline \multicolumn{9}{|l|}{ Control group } \\
\hline C3 & 1 & 1 & 2 & & & & & 4 \\
\hline $\mathrm{C} 4$ & & 3 & 2 & & & & & 5 \\
\hline C5 & & & 8 & & & & & 8 \\
\hline C6 & & & & 1 & & & & 1 \\
\hline $\mathrm{C} 7$ & & & & & 2 & & & 2 \\
\hline Total & 1 & 4 & 12 & 1 & 2 & 0 & 0 & 20 \\
\hline \multicolumn{9}{|l|}{ rTMS group } \\
\hline C3 & 3 & & & & & & & 3 \\
\hline C4 & & 5 & & & & & 1 & 6 \\
\hline C5 & & & 7 & & & & & 7 \\
\hline C6 & & & & 1 & & 1 & & 2 \\
\hline $\mathrm{C} 7$ & & & & & & 1 & & 1 \\
\hline Total & 3 & 5 & 7 & 1 & 0 & 2 & 1 & 19 \\
\hline
\end{tabular}

NLI, neurological level of injury; rTMS, repetitive transcranial magnetic stimulation. 
habilitation, electrical stimulation, magnetic stimulation or their combination have been suggested as strategies in previous reports [23-26].

rTMS has several advantages like non-invasiveness, painlessness, and applicability to severely disabled patients who cannot perform the task-specific exercise. Some neurological disorders including stroke $[27,28]$, Parkinson disease [29], multiple sclerosis [30], and SCI [31-33] have been treated with rTMS, and a few neurological and functional improvements have been reported until date [34]. The exact treatment mechanism of rTMS in neurological disorders is still unclear, but some possibilities involving the following mechanisms have been suggested: synaptic plasticity via long-term potentiation and depression; neurotrophic effects including those of brain-derived neurotrophic factor and glutamate; anti-apoptotic effects, and magnetic-field-induced biophysiological effects $[35,36]$. Previous studies have reported some differences in neurological and functional improvement in SCI patients subsequent to application of rTMS. For example, rTMS at $5 \mathrm{~Hz}$ was not helpful for the improvement of neurological status; functional improvement was seen only in 2 of 15 chronic SCI patients 2 weeks after treatment [32], higher-frequency (10 Hz) rTMS enhanced the neurological and functional improvement in 4 chronic SCI patients 3 weeks after treatment [33], and even though 20 sessions of high-frequency $(20 \mathrm{~Hz})$ rTMS treatment before robot-assisted gait training might have improved the gait function in subacute or chronic SCI patients just after treatment, the observed neurological improvement was not different between sham and rTMS-treated groups [31]. The relationship between the frequency of rTMS and functional improvement after SCI is not clear due to the small number of clinical trials; moreover, variations in the persistence of the therapeutic effect have been noted in different studies. Previous studies have reported that high-frequency $(10 \mathrm{~Hz})$ rTMS can induce long-lasting plasticity of excitatory postsynapses in an animal slice culture system [37] and could be more helpful in improving the grip strength in stroke patients than low-frequency (1 Hz) rTMS [38]. However, previous clinical trials on rTMS treatment of SCI patients involved follow-up of patients only for 2-4 weeks after treatment [32,33]; therefore, it cannot be claimed that the long-term effects of rTMS treatment have been identified clinically. It was observed that for a fine motor task, writing time and score were improved on the treated side in rTMS-treated patients, whereas the improvement in grip and pinch strength was similar in both the treated and non-treated sides (Table 3, Fig. 1). The recovery patterns for dexterity and hand strength might differ according to the complexity of movement, which, in cases of unilateral stroke, is dominated by two or more pathways on both the sides [39]. Nonetheless, a direct comparison was not possible in the present case, as we performed rTMS application unilaterally to bilaterally injured CCS patients. In this study, sensory score change of upper extremity was not apparent in the rTMS treatment group or control group, unlike motor score. Although a previous study reported a partial change in the sensory score 3 weeks after rTMS treatment [33], the 4 -week follow-up period was not adequate for delineation of overall motor and sensory changes. We did not observe any further effects on NLI or AIS improvement in rTMS-treated patients beyond the control in this study; however, previous studies have shown different results. A study, including a sham group, reported no effect of rTMS treatment on AIS improvement [32], whereas the effect of rTMS on neurological or functional recovery was apparent in combination with robot gait training [31] or in non-control studies [33]. All the subjects in the present study were CCS, and most of the initial AIS were D (84.2\% of rTMS-treated patients; $90 \%$ of controls); therefore, small changes in neurological or functional status on the treated side might not be sufficient to change the neurological injury level or severity of both the sides in CCS. No incidences of adverse effects were observed during rTMS treatment or the follow-up period. Previous studies have reported some minor adverse effects of rTMS, including headache, seizure, scalp pain, and facial twitching during rTMS application $[29,40]$.

The present study has several limitations. First, we did not perform sham rTMS treatment for the control group, as the subjects were not willing to undergo the treatment. Therefore, we cannot rule out any bias that might have arisen from the lack of a sham operation or randomization. Second, the follow-up period was only 5 weeks from initial assessments, which is not long enough for detection of any long-lasting effects of rTMS treatment.

In conclusion, our findings suggest that rTMS treatment of SCI patients, especially those with CCS, can enhance motor recovery of upper extremity and some functional 
fine motor task performance on the treated side.

\section{CONFLICT OF INTEREST}

No potential conflict of interest relevant to this article was reported.

\section{ACKNOWLEDGMENTS}

This research was supported by grants (No. NRF2015R1A2A1A15053883 and NRF-2015R1D1A1A02061196) from the National Research Foundation of Korea (NRF) and the Korea Health Technology R\&D Project (No. HI14C0522) by the Ministry of Health \& Welfare, Republic of Korea.

\section{REFERENCES}

1. Ahuja CS, Fehlings M. Concise Review: Bridging the gap: novel neuroregenerative and neuroprotective strategies in spinal cord injury. Stem Cells Transl Med 2016;5:914-24.

2. Nagoshi N, Okano H. Applications of induced pluripotent stem cell technologies in spinal cord injury. J Neurochem 2017;141:848-60.

3. Hong JY, Lee SH, Lee SC, Kim JW, Kim KP, Kim SM, et al. Therapeutic potential of induced neural stem cells for spinal cord injury. J Biol Chem 2014;289:32512-25.

4. Harvey AR, Lovett SJ, Majda BT, Yoon JH, Wheeler LP, Hodgetts SI. Neurotrophic factors for spinal cord repair: which, where, how and when to apply, and for what period of time? Brain Res 2015;1619:36-71.

5. Joo NY, Knowles JC, Lee GS, Kim JW, Kim HW, Son YJ, et al. Effects of phosphate glass fiber-collagen scaffolds on functional recovery of completely transected rat spinal cords. Acta Biomater 2012;8:1802-12.

6. Fuhrmann T, Anandakumaran PN, Shoichet MS. Combinatorial therapies after spinal cord injury: how can biomaterials help? Adv Healthc Mater 2017;6:1601130.

7. Hollis ER 2nd, Ishiko N, Yu T, Lu CC, Haimovich A, Tolentino $\mathrm{K}$, et al. Ryk controls remapping of motor cortex during functional recovery after spinal cord injury. Nat Neurosci 2016;19:697-705.

8. Filli L, Engmann AK, Zorner B, Weinmann O, Moraitis T, Gullo M, et al. Bridging the gap: a reticulo-propriospinal detour bypassing an incomplete spinal cord injury. J Neurosci 2014;34:13399-410.

9. Liu ZH, Yip PK, Adams L, Davies M, Lee JW, Michael GJ, et al. A single bolus of docosahexaenoic acid promotes neuroplastic changes in the innervation of spinal cord interneurons and motor neurons and improves functional recovery after spinal cord injury. J Neurosci 2015;35:12733-52.

10. Siegel CS, Fink KL, Strittmatter SM, Cafferty WB. Plasticity of intact rubral projections mediates spontaneous recovery of function after corticospinal tract injury. J Neurosci 2015;35:1443-57.

11. Hallett M. Transcranial magnetic stimulation: a primer. Neuron 2007;55:187-99.

12. Ellaway PH, Vasquez N, Craggs M. Induction of central nervous system plasticity by repetitive transcranial magnetic stimulation to promote sensorimotor recovery in incomplete spinal cord injury. Front Integr Neurosci 2014;8:42.

13. Jette F, Cote I, Meziane HB, Mercier C. Effect of singlesession repetitive transcranial magnetic stimulation applied over the hand versus leg motor area on pain after spinal cord injury. Neurorehabil Neural Repair 2013;27:636-43.

14. Kumru H, Murillo N, Samso JV, Valls-Sole J, Edwards D, Pelayo R, et al. Reduction of spasticity with repetitive transcranial magnetic stimulation in patients with spinal cord injury. Neurorehabil Neural Repair 2010;24:435-41.

15. Boldt I, Eriks-Hoogland I, Brinkhof MW, de Bie R, Joggi D, von Elm E. Non-pharmacological interventions for chronic pain in people with spinal cord injury. Cochrane Database Syst Rev 2014:CD009177.

16. McKinley W, Santos K, Meade M, Brooke K. Incidence and outcomes of spinal cord injury clinical syndromes. J Spinal Cord Med 2007;30:215-24.

17. van Middendorp JJ, Pouw MH, Hayes KC, Williams R, Chhabra HS, Putz C, et al. Diagnostic criteria of traumatic central cord syndrome. Part 2. A questionnaire survey among spine specialists. Spinal Cord 2010;48:657-63.

18. Fehlings MG, Tetreault L, Nater A, Choma T, Harrop J, Mroz T, et al. The aging of the global population: the changing epidemiology of disease and spinal disorders. Neurosurgery 2015;77 Suppl 4:S1-5.

19. Schneider RC, Cherry G, Pantek H. The syndrome of acute central cervical spinal cord injury; with special 
reference to the mechanisms involved in hyperextension injuries of cervical spine. J Neurosurg 1954; 11:546-77.

20. Mathiowetz V, Weber K, Volland G, Kashman N. Reliability and validity of grip and pinch strength evaluations. J Hand Surg Am 1984;9:222-6.

21. Oudega M, Perez MA. Corticospinal reorganization after spinal cord injury. J Physiol 2012;590:3647-63.

22. Nudo RJ. Rehabilitation: boost for movement. Nature 2015;527:314-5.

23. Fouad K, Tetzlaff W. Rehabilitative training and plasticity following spinal cord injury. Exp Neurol 2012; 235:91-9.

24. Girgis J, Merrett D, Kirkland S, Metz GA, Verge V, Fouad K. Reaching training in rats with spinal cord injury promotes plasticity and task specific recovery. Brain 2007;130:2993-3003.

25. Fujiwara T, Tsuji T, Honaga K, Hase K, Ushiba J, Liu M. Transcranial direct current stimulation modulates the spinal plasticity induced with patterned electrical stimulation. Clin Neurophysiol 2011;122:1834-7.

26. Hou J, Nelson R, Nissim N, Parmer R, Thompson FJ, Bose P. Effect of combined treadmill training and magnetic stimulation on spasticity and gait impairments after cervical spinal cord injury. J Neurotrauma 2014;31:1088-106.

27. Massie CL, Tracy BL, Malcolm MP. Functional repetitive transcranial magnetic stimulation increases motor cortex excitability in survivors of stroke. Clin Neurophysiol 2013;124:371-8.

28. Kim SY, Shin SB, Lee SJ, Kim TU, Hyun JK. Factors associated with upper extremity functional recovery following low-frequency repetitive transcranial magnetic stimulation in stroke patients. Ann Rehabil Med 2016;40:373-82.

29. Brys M, Fox MD, Agarwal S, Biagioni M, Dacpano G, Kumar P, et al. Multifocal repetitive TMS for motor and mood symptoms of Parkinson disease: A randomized trial. Neurology 2016;87:1907-15.

30. Elzamarany E, Afifi L, El-Fayoumy NM, Salah H, Nada M. Motor cortex rTMS improves dexterity in relapsing-remitting and secondary progressive multiple sclerosis. Acta Neurol Belg 2016;116:145-50.

31. Kumru H, Benito-Penalva J, Valls-Sole J, Murillo N, Tormos JM, Flores C, et al. Placebo-controlled study of rTMS combined with Lokomat gait training for treatment in subjects with motor incomplete spinal cord injury. Exp Brain Res 2016;234:3447-55.

32. Kuppuswamy A, Balasubramaniam AV, Maksimovic R, Mathias CJ, Gall A, Craggs MD, et al. Action of $5 \mathrm{~Hz}$ repetitive transcranial magnetic stimulation on sensory, motor and autonomic function in human spinal cord injury. Clin Neurophysiol 2011;122:2452-61.

33. Belci M, Catley M, Husain M, Frankel HL, Davey NJ. Magnetic brain stimulation can improve clinical outcome in incomplete spinal cord injured patients. Spinal Cord 2004;42:417-9.

34. Lefaucheur JP, Andre-Obadia N, Antal A, Ayache SS, Baeken C, Benninger DH, et al. Evidence-based guidelines on the therapeutic use of repetitive transcranial magnetic stimulation (rTMS). Clin Neurophysiol 2014;125:2150-206.

35. Chervyakov AV, Chernyavsky AY, Sinitsyn DO, Piradov MA. Possible mechanisms underlying the therapeutic effects of transcranial magnetic stimulation. Front Hum Neurosci 2015;9:303.

36. Soundara Rajan T, Ghilardi MFM, Wang HY, Mazzon E, Bramanti P, Restivo D, et al. Mechanism of action for rTMS: a working hypothesis based on animal studies. Front Physiol 2017;8:457.

37. Vlachos A, Muller-Dahlhaus F, Rosskopp J, Lenz M, Ziemann U, Deller T. Repetitive magnetic stimulation induces functional and structural plasticity of excitatory postsynapses in mouse organotypic hippocampal slice cultures. J Neurosci 2012;32:17514-23.

38. Sasaki N, Mizutani S, Kakuda W, Abo M. Comparison of the effects of high- and low-frequency repetitive transcranial magnetic stimulation on upper limb hemiparesis in the early phase of stroke. J Stroke Cerebrovasc Dis 2013;22:413-8.

39. Noskin O, Krakauer JW, Lazar RM, Festa JR, Handy C, O'Brien KA, et al. Ipsilateral motor dysfunction from unilateral stroke: implications for the functional neuroanatomy of hemiparesis. J Neurol Neurosurg Psychiatry 2008;79:401-6.

40. Rossi S, Hallett M, Rossini PM, Pascual-Leone A; Safety of TMS Consensus Group. Safety, ethical considerations, and application guidelines for the use of transcranial magnetic stimulation in clinical practice and research. Clin Neurophysiol 2009;120:2008-39. 